\title{
Improved Random Channel Access for OFDMA Wireless Networks
}

\author{
G. Das, B. Lannoo, D. Colle, M. Pickavet, P. Demeester
}

\begin{abstract}
In this paper we propose a new random channel access protocol to reduce the channel access delay in a multichannel orthogonal frequency division multiple access (OFDMA) wireless networks. In particular, we propose a two dimensional random walk through time and frequency domain slots simultaneously to reduce the random channel access delay for the IEEE 802.16e-2005 based WiMAX media access control (MAC) protocol. We prove through simulation that our proposed scheme improves upon the existing random channel access mechanism with truncated binary back-off, currently implemented in WiMAX. We demonstrated that with our proposed channel access mechanism, a mobile node with real time traffic requirements will have minimum service disruption due to the reduced delay in the initial ranging and the bandwidth request phase while changing rooms.
\end{abstract}

\section{Index Terms-WiMAX, OFDMA, Random Channel Access}

\section{INTRODUCTION}

$\mathrm{T}$ IEEE 802.16e-2005 World Interoperability for Microwave Access (WiMAX) supports both fixed and mobile point to multipoint networks, and it has already promised to be one of the key metropolitan area networks [1, 2]. The WiMAX standard recommends implementing the orthogonal frequency division multiple access (OFDMA) technique which has multiple advantages like overcoming multipath fading effect, facilitating better dynamic bandwidth allocation, etc.

Regarding dynamic bandwidth allocation, OFDMA enhances the overall performance by allocating radio resources in both the time and frequency domain. WiMAX uses centralized multipoint to central point MAC protocol, where a centralized base station (BS) coordinates the data transmission from various mobile stations (MS). In WiMAX, all the MSs transmit data directly to the BS in the uplink direction. The BS then distributes data among the MSs for the local traffic and forward external traffic to the respective MSs in the downlink direction. The downlink data in WiMAX is generally broadcast to all MSs and the respective MS filters out its data from its destination address. However, in the uplink direction WiMAX requires a MAC protocol to coordinate the multipoint to central point (BS) transmission. The WiMAX standard supports both a connection oriented

G. Das, B. Lannoo, D. Colle, M. Pickavet, P. Demeester are with Dept. of Information Technology (INTEC), Ghent University - IBBT, Gaston Crommenlaan 8 box 201, 9050 Ghent, Belgium. (e-mail: goutam.das@intec.ugent.be). and contention based protocol for channel access in the uplink direction. The purpose of a connection oriented MAC protocol is to allow MSs to transmit data to the BS in the reserved time slots and sub-channels of the OFDM physical channel. However, MSs have to request for channel access to the BS prior to actual data transmission. This process is facilitated in WiMAX through contention based MAC, where a few of the uplink sub-channels are reserved for this purpose. WiMAX contention based MAC employs a multi-channel random access protocol with binary back-off to avoid and resolve contention. However we show in our paper that this channel random access protocol introduces a higher channel access delay in high load conditions.

Therefore, in this paper we propose a new algorithm for channel access mechanism and through simulation we show that our proposal reduces the overall channel access delay significantly. The rest of the paper is organized as follows. Section II provides a brief introduction to the WiMAX channel access protocol, together with a brief summary of the other existing algorithms for random channel access from the literature. Section III introduces the motivation behind our problem statement and the relevance of its requirement. Finally this section describes our proposed algorithm in further detail. Section IV discusses the results and their interpretation and finally Section $\mathrm{V}$ concludes the paper.

\section{WIMAX MAC PROTOCOL FOR RANDOM CHANNEL ACCESS}

In this section we provide a brief overview of the WiMAX MAC protocol and list the different random channel access mechanisms that have been proposed in the literature.

\section{A. Brief overview of the WiMAX MAC protocol}

As described in the previous section WiMAX employs a connection oriented as well as contention based MAC protocol for uplink data transmission. Fig. 1 provides the timing illustration of the WiMAX OFDMA based MAC protocol for up and down link data transmission. WiMAX uses the same OFDMA channels to transmit both up and downlink data. As shown in Fig. 1 up and downlink transmissions are subdivided into sub-frames and both up and downlink sub-frames are mutually interleaved. The downlink data frame contains downlink map (DL-MAP) and uplink map (UL-MAP) to indicate all the MSs about the time and sub-channel to pick for downlink data reception and uplink data transmission respectively. In WiMAX all the users in the previous uplink 


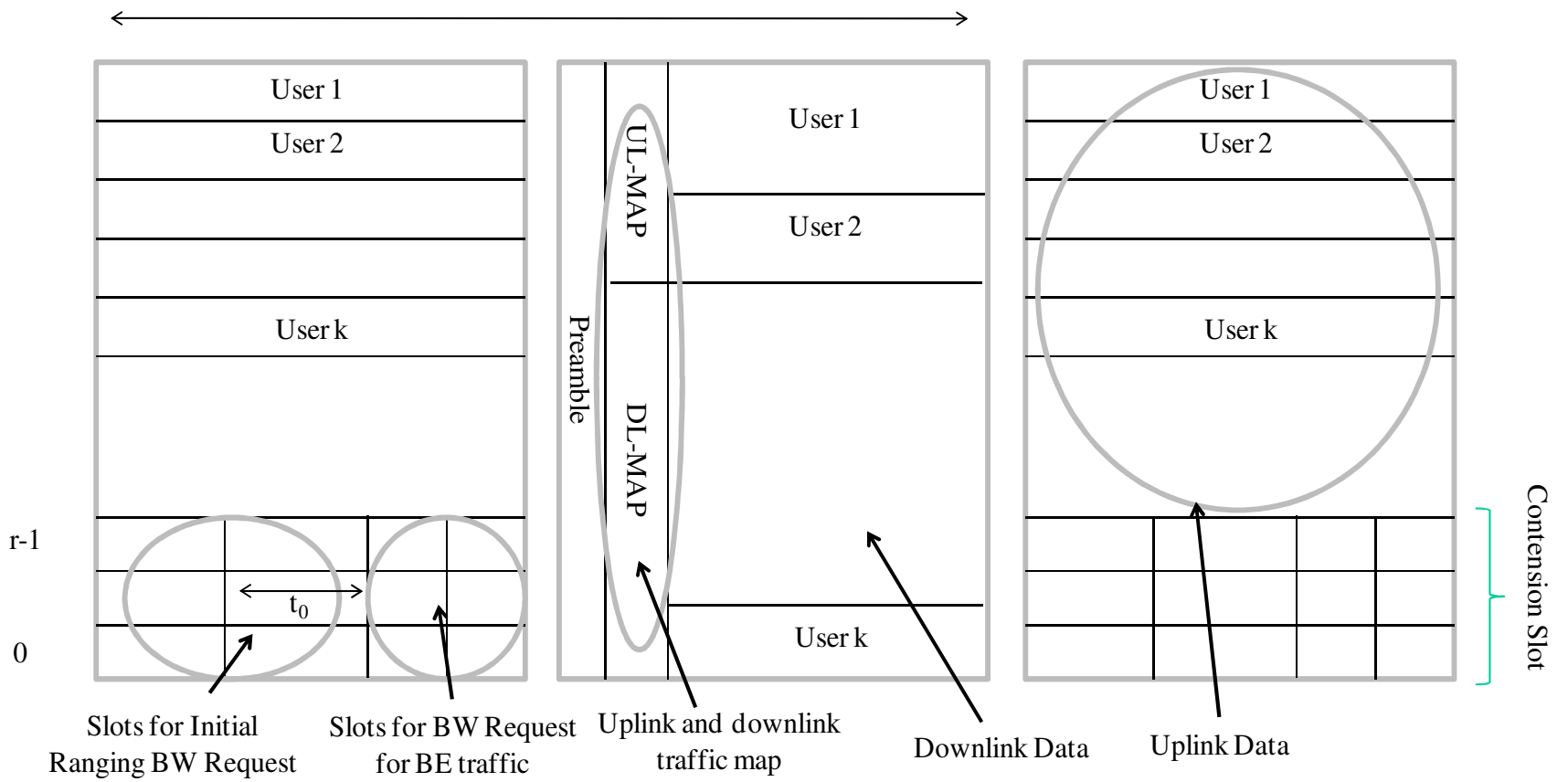

Fig. 1: Frame Structure of IEEE 802.16e.

frame request for additional data transmission slots to the BS for the next uplink sub-frame. The BS runs a scheduling algorithm to allocate OFDMA sub-channels and time slots to the MSs according to their needs. Corresponding UL-MAP is issued by the BS just at the beginning of the next downlink sub-frame to notify the MSs about their respective allocation. Simultaneously, downlink traffic for different MSs is scheduled and notified through the DL-MAP. In WiMAX the MS informs about its traffic requirement for the $(n+1)^{\text {th }}$ uplink sub-frame to the BS within the $n^{\text {th }}$ downlink sub-frame through piggybacking. Therefore, the MSs that are already registered with a BS can send their uplink data request without being involved in the random channel access phase. The MSs that are new to the network or that have experienced hand-off recently or that are registered with the concerned BS but have suffered loss of some specified number of successive uplink transmission, can participate in the random channel access phase. There are two random access phases defined in WiMAX as shown in Fig. 1. The first one is used for the initial ranging and channel access for the new or disconnected users. The second random channel access phase is used for notifying the bandwidth demand to the BS for particularly the best effort traffic or for MSs that have been successful in the initial ranging phase. The time slot available for the initial ranging is much longer than the slot duration of the best effort traffic request. This is due to the long preamble required for synchronization. Generally any connection request in WiMAX, be it real time of best effort, has first to contend for channel access for the initial ranging phase. Also a disconnected node, either due to the mobility and associated hand-off or due to channel fading, might have to contend for the initial ranging phase all over again. Therefore for real time traffic the initial ranging delay might prove to be detrimental if not being controlled properly.

In this paper, our major focus is to improve the contention based MAC for the initial ranging and bandwidth (BW) request phase as well as the bandwidth request phase for the best effort traffic as shown in Fig. 1. This will also help in reducing the overall delay performance for the best effort traffic as well. We assume that there are $r$ OFDMA subchannels allocated for this purpose. Each of the sub-channels is further subdivided into multiple time slots of equal length. WiMAX adopts a multi-channel random access protocol with binary back-off for collision avoidance [3], where each MS initially choose a contention window $(\mathrm{CW})$ of length $W_{0}$ and further selects a random number $(x)$ from 0 to $W_{0^{-}}-1$. The MS, that wants to transmit a BW request to the $\mathrm{BS}$, waits for $x$ amount of time slots before transmitting the BW request packet. While transmitting, the MS selects randomly an OFDMA sub-channel among $r$ and transmits. If collision occurs, the $\mathrm{CW}$ is increased to $2 W_{0}$. With further collisions, the contention window size follows a truncated binary backoff increment. Therefore, after $i$ successive collisions the CW size becomes $2^{i} W_{0}$. The process continues till the $m^{\text {th }}$ collision. Beyond this, if collision occurs, the $\mathrm{CW}$ is fixed to $2^{m} W_{0}$.

\section{B. Related work}

Several previous studies have been reported in the literature for the different multi-channel random access protocols for the WiMAX radio subsystem. In [4], a new random access 
algorithm was proposed and analyzed, where a fast retrial algorithm is imposed upon the MSs to choose a random OFDMA sub-channel immediately for data retransmission instead of adopting time back-off for the first few collisions. Thereafter, if the retransmission number increases beyond some predefined threshold (retransmission threshold $M$ ) the MS starts backing of in time. This imposes a natural problem to the algorithm as the design parameter $M$ has to be optimally designed. As with high load, if $M$ is very low the MSs will go into time back-off very quickly and will introduce delay. On the contrary if $M$ is high, the collisions will not be resolved till the retry number reaches the value $M$ and therefore channel utilization will be poor for longer duration. In [3], the authors have proposed and analyzed the multi-channel random access with truncated binary back-off as described in the previous sub-section. However, binary back-off has the problem of increasing the time domain collision window too fast (exponential) and hence introducing more delays. In [5-6], the authors have proposed a new channel random access, however, that is applicable for single carrier radio WiMAX and in this paper we mainly focus on multi-channel OFDMA random access. Therefore, the proposal of [5-6] is not considered in the context of this paper.

In the next section we propose a novel and simple method to remedy the channel access delay problem present with the existing WiMAX protocol and its other variation from the literature. Our current analysis mainly focuses on the initial ranging phase. We keep the detailed study of the bandwidth request phase for the best effort traffic for future studies. However, as the random access processes are similar for both phases, the analysis and conclusion in this paper are also valid for the best effort traffic.
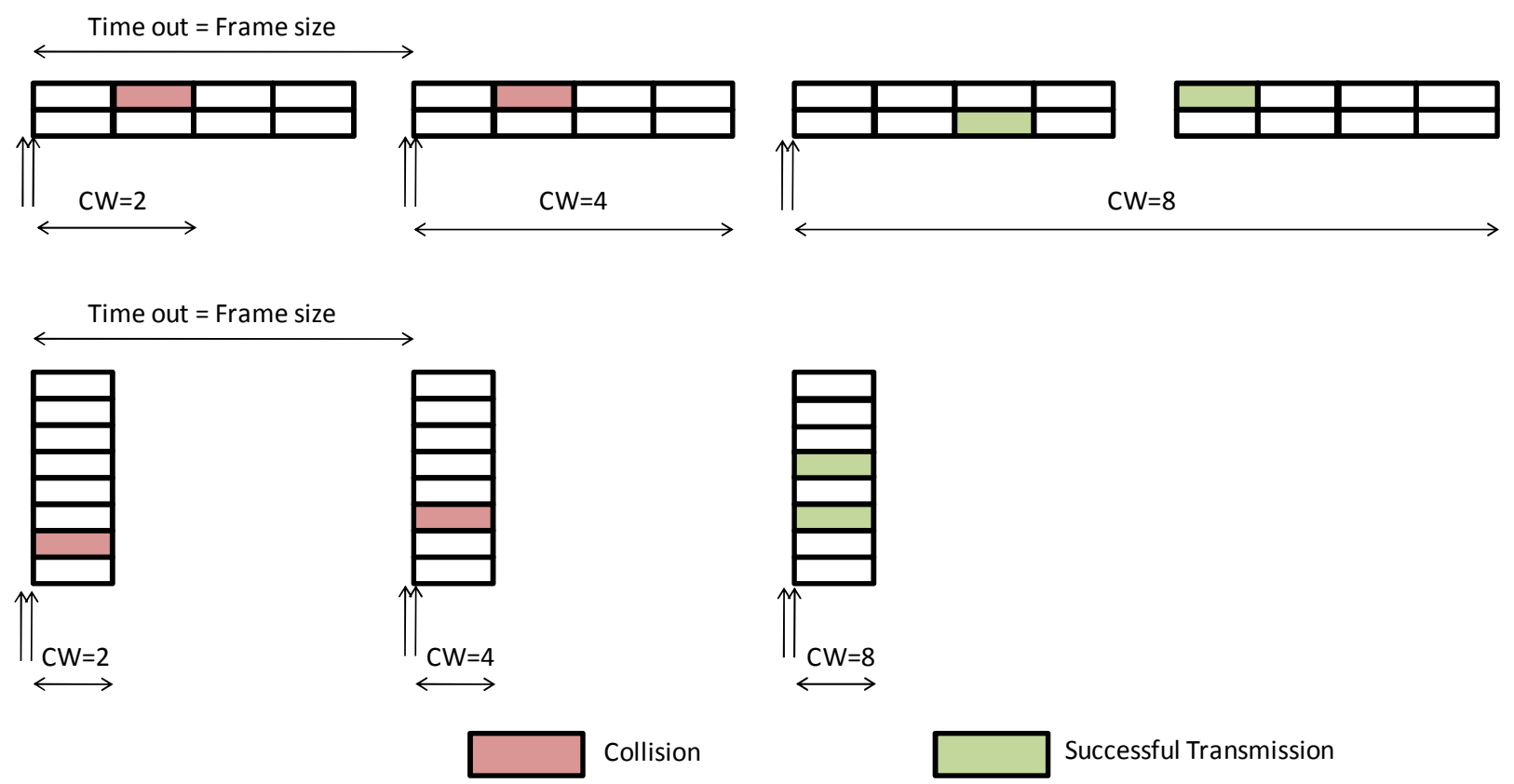

\section{PRoposed RANDOM ChanNel ACCESS DElay}

To motivate the requirement for the modification of the existing WiMAX multi-channel random access protocol with truncated binary back-off we introduce an example illustration shown in Fig. 2. In particular, Fig. 2 compares two different scenarios with the first one having two OFDMA sub-carriers reserved for the contention resolution and the second one having 8 OFDMA sub-carriers reserved for the contention resolution. However both cases have 8 time-frequency combined slots available for the initial ranging and bandwidth allocation. For simplicity we further assume that the frame length and the time out period are equal in length. We assume for both the cases, two stations have an initial ranging request simultaneously and both of them get their collision resolved after two successive retransmissions. Fig. 2 clearly depicts that the delay will be reduced in the second case. The first case depicts the existing WiMAX protocol using the multi-channel random access with binary back-off. In the second case we use a modified back-off algorithm that runs the back-off algorithm in a combined time and frequency domain compared to the binary back-off for existing WiMAX which only uses the time slot domain for increasing the back-off (as shown in the first

In our proposal the node requiring random access, walks through the two-dimensional (i.e. time and frequency) slot map as shown in Fig. 3. In this process, time slots are repeated as many times as the number of available frequencies ( $r$ for our case). In fact we run the back-off algorithm with an increasing collision window on that derived repeated slot map instead of running it in the actual time (i.e. one-dimensional) as shown in Fig. 3. Every time a user detects a collision (not getting an acknowledgement packet (ACK) for the previous case of the illustration of Fig. 2).

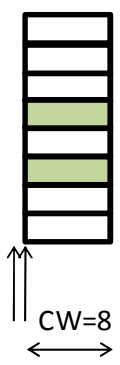

Successful Transmission

Fig. 2: A simplified example illustration for demonstrating the problem with the current WiMAX random channel access. 

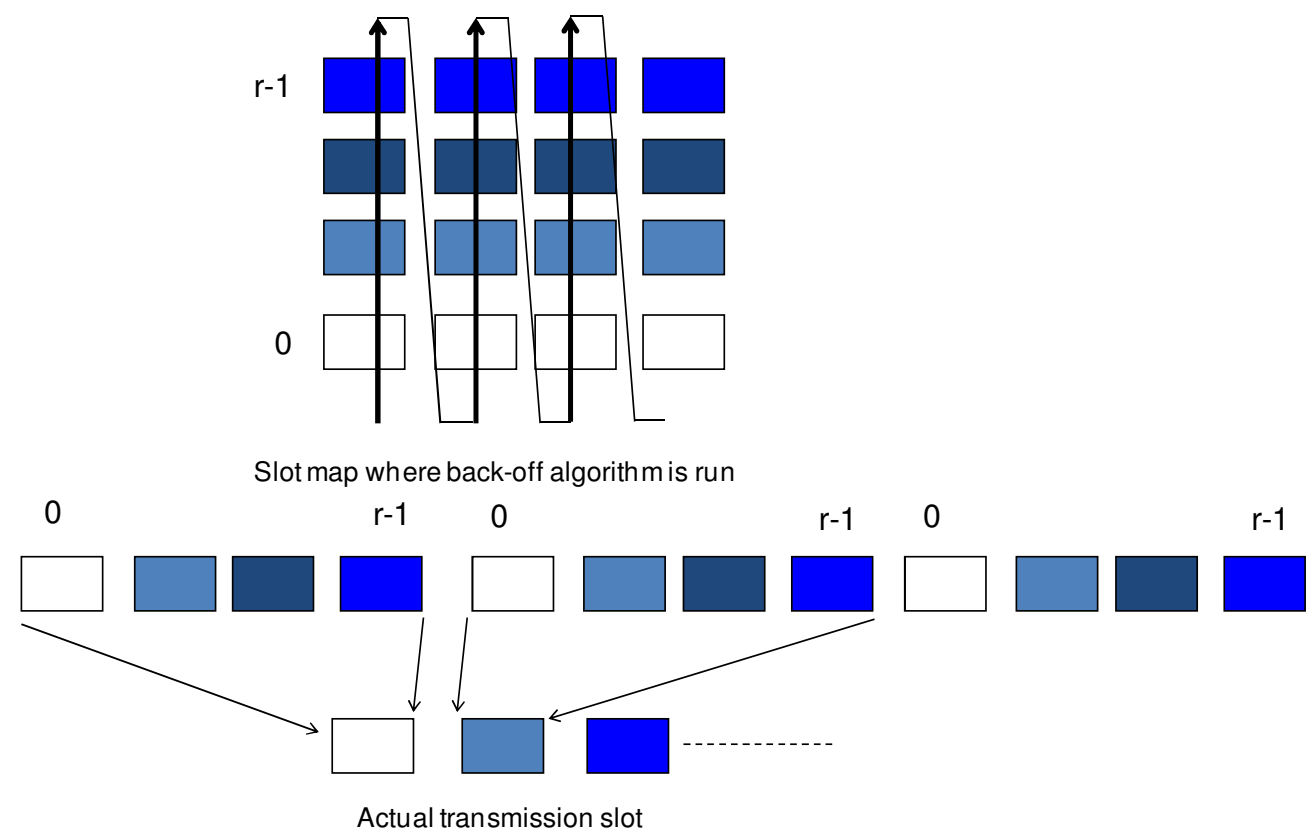

Fig. 3: Two-dimensional random back-off algorithm.

attempt after a timeout) it increases its collision window following the back-off algorithm and goes for channel access in the next available time slot. In that time slot, it starts counting from the lowest frequency slot available and generates a random number $\mathrm{x}$ from 0 to $r$ - 1 . The users start their collision window from carrier slot $\mathrm{x}$ and generates another random number y between 0 and current collision window. It then walks through the combined time and carrier slot map shown in Fig. 3 to wait for y numbers of slots before acquiring a slot and attempting transmission. In Fig. 3 different colors depict different OFDMA carriers. This process reduces the delay in the collision avoidance process significantly when many users contend for channel access as shown with the example in Fig. 2. From this illustration, it is also evident that for higher loads we need to include the maximum number of OFDMA carriers possible into account for contention resolution in the random channel phase. This means that number of OFDMA carriers $(r)$ has to be much larger than 1 while operating in the high load and hence higher contention prone operation mode.

Fig. 4 provides an example illustrating the execution of the back-off algorithm described above. Here we have taken $r=4$. At the end of the second time slot, a user tries to access the wireless channel with a collision window 4 . It first generates a random number $\mathrm{x}$. We assumed $\mathrm{x}$ to be 2 . The user starts $\mathrm{CW}$ count after $\mathrm{x}$, as shown in Fig. 4. The user then generates another random number y between 0 and $\mathrm{CW}-1$ (we assumed $y=1$ for our example) and waits for y more slots before accruing the channel as shown in Fig. 4.

From Fig. 2, 3 and 4 it is easy to understand that as the number of OFDMA carriers reserved for channel access $(r)$ increases, the channel access delay gets reduced. Therefore, it is advisable to use all the OFDMA carriers or a maximum number of OFDMA carriers while keeping the number of combined time-frequency slots constant. This is to ensure that the total percentage of bandwidth reserved for contention resolution remains the same. We will further provide simulation evidence to prove our claim (illustrated with the example in this section) of delay improvement in the next section.

This concludes the description of our improved multichannel random access scheme for WiMAX initial ranging and bandwidth request. In the next section we provide simulation results to show the improvement of our proposal compared to the existing WiMAX channel access scheme.

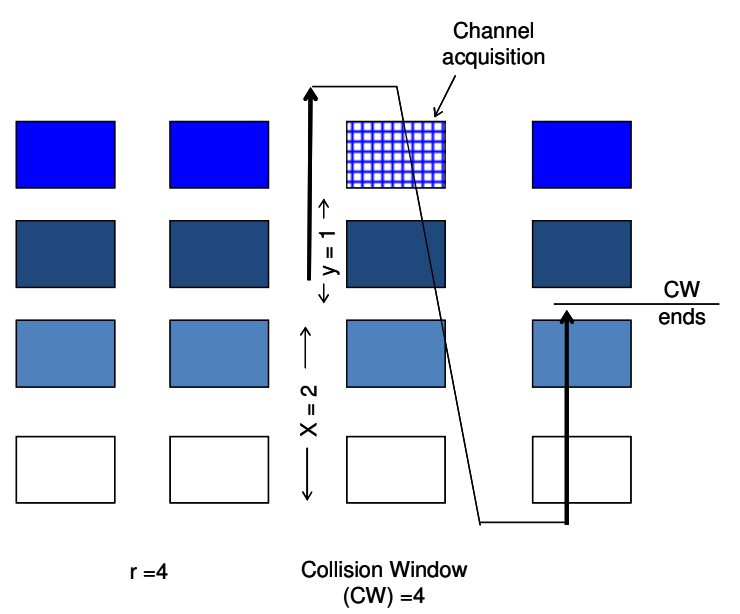

Fig. 4: An example of the execution of the proposed back-off algorithm. 


\section{RESULTS AND DISCUSSION}

In this section we provide the numerical results to prove the effectiveness of our proposed algorithm in reducing the channel access delay. For the numerical analysis, we assume the following parameters:

$\begin{array}{ll}\text { Frame Duration }\left(T_{f}\right) & 2 \mathrm{msec} \\ \text { Timeout Duration }\left(T_{0}\right) & 10 \mathrm{msec} \\ \text { Initial back-off Window }\left(W_{0}\right) & 4 \\ \text { Final back-off Window }\left(W_{m}\right) & 64 \\ \text { Ranging slot duration }\left(t_{0}\right) & 50 \mu \mathrm{sec} \\ \text { Number of reserved time slot }(m) & 1,16,32 \\ \text { Number of OFDMA sub channels } & \\ \text { for initial ranging }(r) & 64,4,2\end{array}$

The example values of $m$ and $r$ are chosen to demonstrate that the proposed algorithm provides the benefits that have been described in the previous section. Therefore, varying the values of $m$ and $r$ will not affect the general conclusion of the paper.

Fig. 5 shows the plot for the delay in function of the offered load for different combinations of the OFDMA carrier number $(r)$ and time slot number $(m)$ within a frame. Note that the offered load for random access is calculated as the number of access requests per frame divided by the total number of slots available per frame for ranging $(r \times m=64)$. E.g. a load of $33 \%$ corresponds to 22 access requests per frame. For the case with $r=64$ and $m=1$, we use our proposed modified algorithm. In the other two cases we use the WiMAX multichannel random access protocol with binary back-off. For fair comparison we use the same number of all combined time and frequency slots per frame so that the bandwidth wastage in the uplink channel remains the same for all the scenarios. We observe (as predicted in the previous section) that in the high load scenario our proposed algorithm reduces the overall channel access delay. This is due to the fact that at the higher load the number of collisions experienced by each packet is increased and therefore running a binary back-off in the time domain with less number of OFDMA carriers increases the delay as depicted in Fig. 2. On the other hand, at lower load, as our proposed MAC solution uses only one time slot per frame, the transmission without collision always happens in the next frame. Therefore, at a lower load our proposed scheme provides more delay than the WiMAX MAC.

Fig. 6 shows the channel utilization by the combined reserved frequency and time slot for initial ranging under the same sets of the test scenario as of Fig. 5. As expected, at the higher load, our proposal provides better channel utilization compared to that of the WiMAX binary back-off algorithm. The interesting observation from Fig. 6 is that almost all the solutions have a definite saturation channel utilization around an offered load of $33 \%$ and the random access protocol cannot provide any better channel utilization than that.

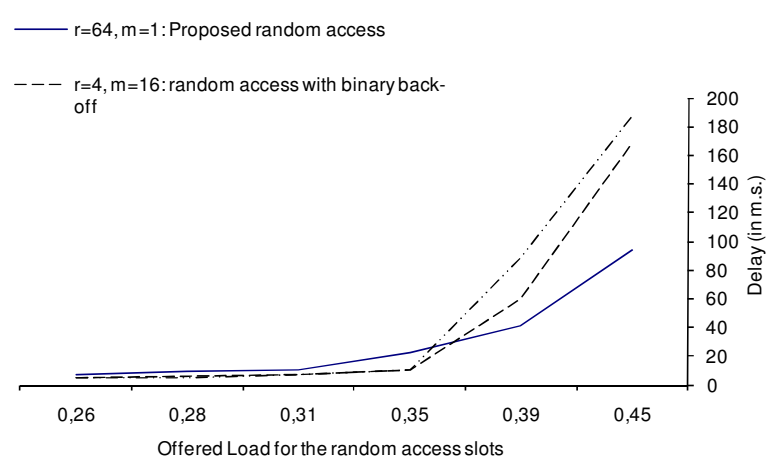

Fig. 5: Delay measurement of WiMAX initial ranging algorithm for different combination of time slot per frame and OFDMA carriers.

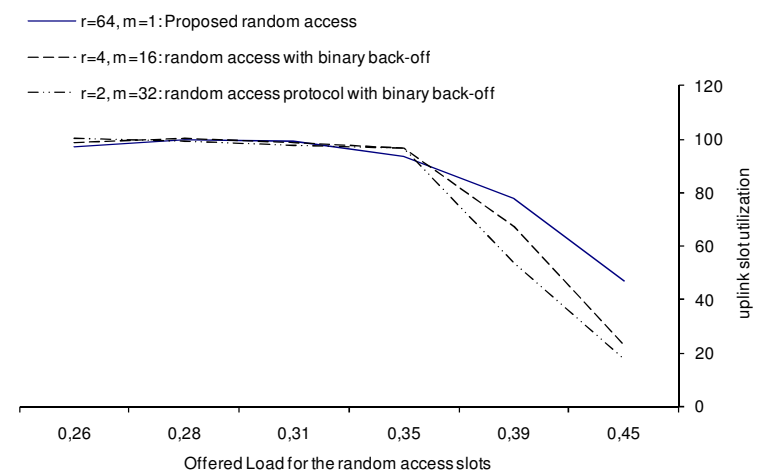

Fig. 6: Channel Utilization for the initial ranging slot with random access load.

Fig. 7 and 8 provide the delay evolution for the test scenario with $r=64$ and $m=1$ with a traffic load of 0.35 and 0.45 respectively. This shows that as the load increases beyond the crossover point, where the link utilization is the maximum, the delay profile starts increasing monotonically and the network operates under saturation.

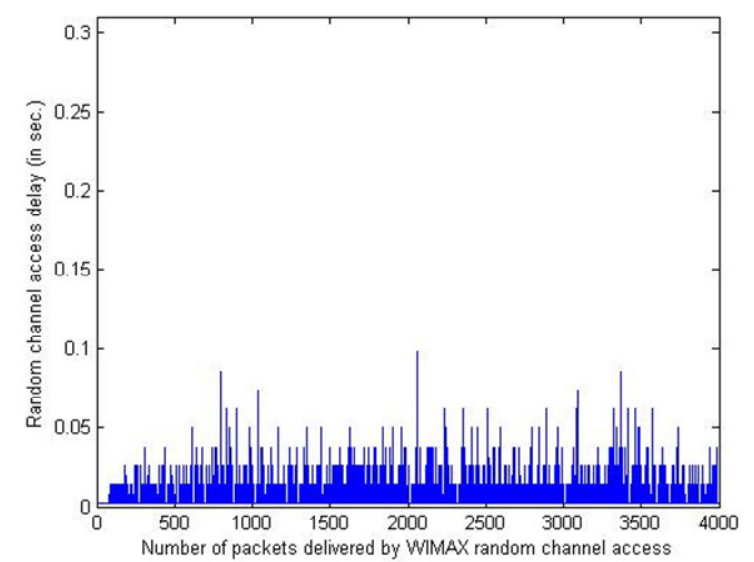

Fig. 7: Channel access delay evolution with packet number (time) in steady state. 


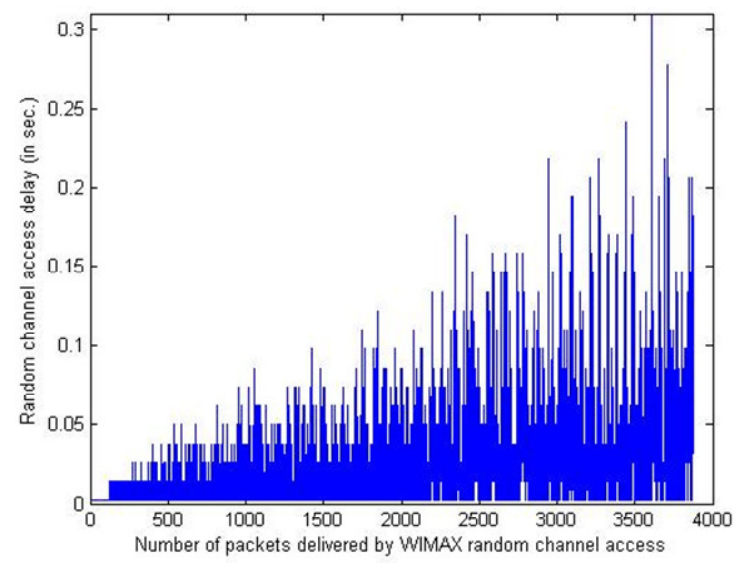

Fig. 8: Channel access delay evolution with packet number (time) with saturation load.

In Fig. 9 we plot the channel access delay with respect to a varying initial window size $\left(W_{0}\right)$ of our proposed back-off algorithm. For $r=64$ and the available combined time and frequency slot equals to 64 , we observe that, our algorithm works best when $W_{0}$ is set to 8 .

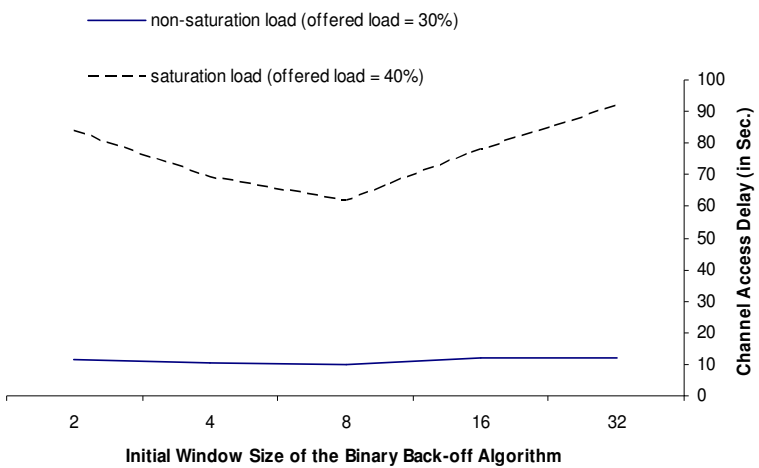

Fig. 9: Channel access delay evolution with the varying initial window size $\left(W_{0}\right)$ for both saturated and non-saturated load condition.

An important point to be noted is that our proposal works much better under the network saturation load and therefore we propose to modify the WiMAX protocol to adapt to our MAC procedure whenever the traffic load increases to the critical limit. We observe that the current WiMAX protocol is better under low load conditions and hence we recommend to keep WiMAX operating with binary back-off in time domain as long as the network load remains under control and to switch over to our proposed scheme once the load increases. This eventually can ensure the stringent delay requirement for real time traffic under heavy load combined with a high mobility scenario.

\section{CONCLUSION}

In this paper we have proposed a new multi-channel random access protocol to reduce the channel access delay of the initial ranging phase compared to the existing WiMAX multichannel random access contention-based MAC protocol with truncated exponential binary back-off algorithm. We proved through simulations that our proposal significantly improves upon the channel access delay values for a typical WiMAX network with high load. This will enable a higher degree of mobility among the WiMAX subscribers demanding stringent delay requirement for real time traffic.

\section{ACKNOWLEDGEMENTS}

The research leading to these results has received funding from the EC in the FP7 ICT-ALPHA project.

\section{REFERENCES}

[1] IEEE std. 802.16-2004, IEEE Standard for local and metropolitan area networks- part 16: Air Interface for fixed broadband wireless access systems, Oct. 2004.

[2] IEEE std. 802.16e-2005, IEEE Standard for local and metropolitan area networks- part 16: Air Interface for fixed broadband wireless access systems amendment for physical and medium access control layers for combined fixed and mobile operation in licensed bands, Dec. 2005.

[3] Li-Chun Wang, A. Chen, " Optimal radio resource partition for joint contention and connection oriented multichannel access in OFDMA systems," in IEEE Transactions on Mobile Computing, Vol. 8, No. 2, February 2009, pp. $162-172$

[4] Y. J. Choi, S. Park, S. Bahk, "Multichannel random access in OFDMA wireless networks," IEEE Journal of Selected Areas in Communications, Vol. 24, March 2006, pp. 603 - 613.

[5] A. Vinel, Q. Ni, D. Staehle, A. Turlikov, "Capacity analysis of reservation based random access for broadband wireless access networks," IEEE Journal of Selected Areas in Communications, Vol. 27, No. 2, February 2009, pp. $172-181$

[6] S. Andreev, Z. Saffer, A. Turlikov, A. Vinel, "Overall delay in IEEE 802.16 with contention-based random access," in Springer Lecture Notes in Computer Science, 2009, Vol. 5513/2009, pp. 89 - 102. 